OPEN ACCESS

Edited by:

Kalijn Bol,

Radboud University Nijmegen Medical

Centre, Netherlands

Reviewed by:

Kathleen Marie Kokolus,

University at Buffalo, United States

Camelia Quek,

Melanoma Institute Australia, Australia

*Correspondence:

Kazuto Tajiri

tajikazu@med.u-toyama.ac.jp

Specialty section:

This article was submitted to

Skin Cancer,

a section of the journal

Frontiers in Oncology

Received: 26 February 2021

Accepted: 10 May 2021

Published: 02 June 2021

Citation:

Takahashi N, Tajiri K, Kagoyana K, Tanaka S and Yasuda I (2021) CD8

Positive T Lymphocyte Infiltration of Liver Metastases of Uveal Melanoma:

A Case Report.

Front. Oncol. 11:672660.

doi: $10.3389 /$ fonc. 2021.672660

\section{CD8 Positive T Lymphocyte Infiltration of Liver Metastases of Uveal Melanoma: A Case Report}

\author{
Naoki Takahashi ${ }^{1}$, Kazuto Tajiri ${ }^{1 *}$, Ko Kagoyana ${ }^{2}$, Shinichi Tanaka ${ }^{3}$ and Ichiro Yasuda ${ }^{1}$ \\ 1 The Third Department of Internal Medicine, Faculty of Medicine, University of Toyama, Toyama, Japan, ${ }^{2}$ Department of \\ Dermatology, Faculty of Medicine, University of Toyama, Toyama, Japan, ${ }^{3}$ Department of Diagnostic Pathology, Faculty of \\ Medicine, University of Toyama, Toyama, Japan
}

A 78-year-old Japanese man was referred for examination of multiple small nodules on his liver detected by magnetic resonance imaging (MRI). These small nodules were hyperintense on T1-weighted MRI, and were in hepatobiliary phase on gadoliniumethoxybenzyl-diethylenetriamine pentaacetic acid enhanced MRI. Five years earlier, he had undergone curative enucleation of his left eye for uveal melanoma. US-guided biopsy revealed that the liver nodules were metastases of melanoma. Pathological examination also showed infiltration of CD8 positive T lymphocytes. The metastatic nodules remained unchanged for more than 2 years and he was not further treated. This pathology and clinical course suggest that the systemic immune response of the host could suppress hepatic metastases of uveal melanoma.

Keywords: malignant melanoma, uveal melanoma, liver metastasis, CD8 positive T lymphocyte, host immune response

\section{INTRODUCTION}

Malignant melanoma is a neoplasm derived from melanocytes. The most common primary site of this neoplasm is the skin, followed by the mucosa and eyes. Malignant melanoma is relatively rare in Asia and the prognosis of patients with metastatic melanoma is extremely poor. Uveal melanoma is a rare disease, with a reported annual incidence of 5.5-6 per million among Whites and 0.2-0.3 per million among Asians (1). Uveal melanoma is aggressive and metastasizes hematogenously, most frequently to the liver (60-87\%), but also to the lungs (24-26\%), skin (10 - 17\%), bones (8.4-29\%), and brain $(4.2 \%)(2-4)$. Liver metastasis is the leading cause of death in patients with malignant melanoma, with early detection of liver metastases important in prolonging survival (5). Regarding with the development of liver metastasis from uveal melanoma, a study with autopsy cases suggested that hepatic micrometastasis can occur in advance clinical diagnosis of the primary tumor (6). The metastatic tumor develops with from infiltrative pattern to nodular growth pattern, and the infiltrating immune cells change from natural killer (NK) cells to CD3-positive lymphocytes according to tumor growth (7). Thus not only adoptive but also innate immune cells including NK cells contribute to the formation of tumor microenvironment (8). However, on the other hand, uveal melanoma is refractory to immunotherapy, the standard treatment for metastatic melanoma, with the median survival of patients diagnosed with liver metastases of uveal melanoma being only several months (9-11). A recent study reported that CD8 positive $\mathrm{T}$ lymphocytes expressing 
lymphocyte activation gene-3, rather than programmed death-1 or cytotoxic T-lymphocyte antigen 4, are involved in immunecheckpoint mechanism in uveal melanoma (12). Further investigation is required to improve the prognosis of uveal melanoma.

We encountered a patient with small liver metastases of uveal melanoma detected 5 years after surgical resection of the primary lesion. Despite a lack of treatment, these small liver metastatic nodules showed no evidence of progression for more than 2 years. Biopsies of the liver nodules showed infiltration of CD8 positive lymphocytes, suggesting that host immunity could suppress the progression of disease, depending on the status of liver metastases.

\section{CASE REPORT}

A 78-year-old Japanese man was referred to our department for examination of multiple small nodules, of diameter 4-5 mm, detected by magnetic resonance imaging (MRI). These small nodules showed high-intensity on T1 weighted MRI (Figure 1A), iso-intensity on T2 weighted imaging (Figure 1B), and high-intensity on hepatobiliary phase of gadolinium- ethoxybenzyl-diethyleneaminepantaacetic acid (Gd-EOBDTPA)-enhanced MRI (Figure 1C). Five years earlier, he had undergone curative enucleation of his left eye for uveal melanoma, but he was asymptomatic during this 5-year period. Laboratory data showed slight increases in the concentrations of hepatobiliary enzymes, including aspartate aminotransferase (32 U/L), alanine aminotransferase $(26 \mathrm{U} / \mathrm{L})$, alkaline phosphatase (383 U/L), $\gamma$-glutamyl transpeptidase (227 U/L), and total bilirubin $(1.0 \mathrm{mg} / \mathrm{dL})$. Serologic examination showed no evidence of hepatitis $\mathrm{B}$ or $\mathrm{C}$ virus infection or autoimmune liver injury. Annual evaluation by fluorodeoxyglucose positron emission tomography (FDG-PET)/computed tomography (CT) showed no evidence of abnormal FDG uptake during this 5-year period. Retrospective examination, however, identified small hyperdense small nodules on the plain phase of FDG-PET/CT at least 1 year earlier (Figures 1D, E). These nodules were not enhanced on dynamic CT (Figures 1F-H) but were hypoechoic on B-mode ultrasonography (US) (Figure 2A). On Sonazoidenhanced US, these nodules showed strong enhancement during early arterial phase (Figure 2B) and were hypoechoic on delayed phase (Figure 2C), suggesting that these nodules were liver metastases of uveal melanoma.Macroscopic examination of US-guided biopsy specimens of the liver nodules showed black
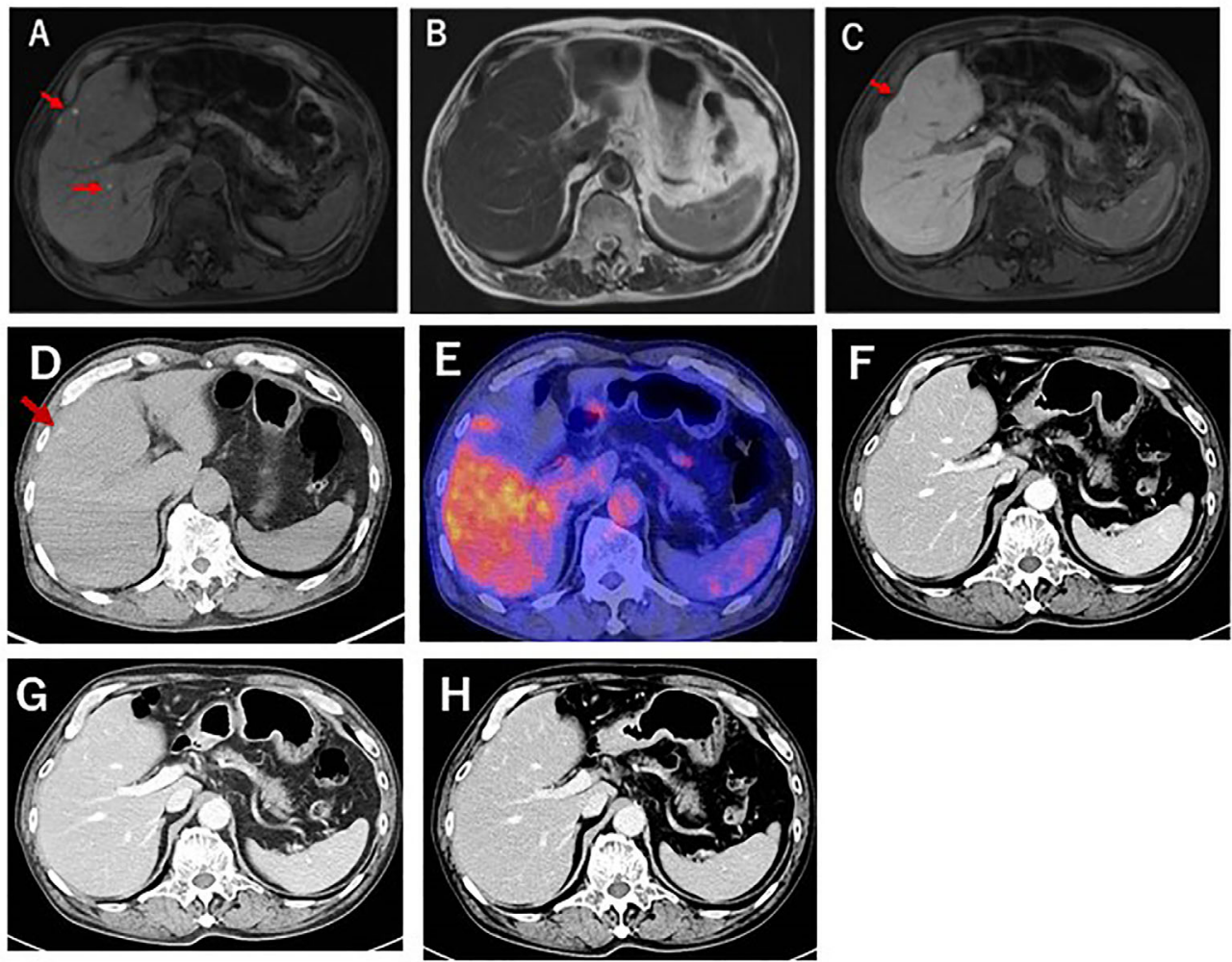

FIGURE 1 | Gd-EOB-DTPA enhanced MRI of this patient. (A) T1-weighted MRI showing that the liver nodules (red arrows) were highly intense. (B) T2-weighted MRI, showing that the liver nodules were iso-intense. (C) Gd-EOB-DTPA-enhanced MRI, showing the showed were highly intense during the hepatobiliary phase. (D) Plain-CT examination 1 year before liver biopsy, showing small, high-density nodules. (E) FDG-PET CT examination at liver biopsy. No abnormal FDG uptake was detected. (F-H) Dynamic CT examination during the (F) arterial, (G) portal phase, and (H) equivalent phases. No enhancement was detected. 

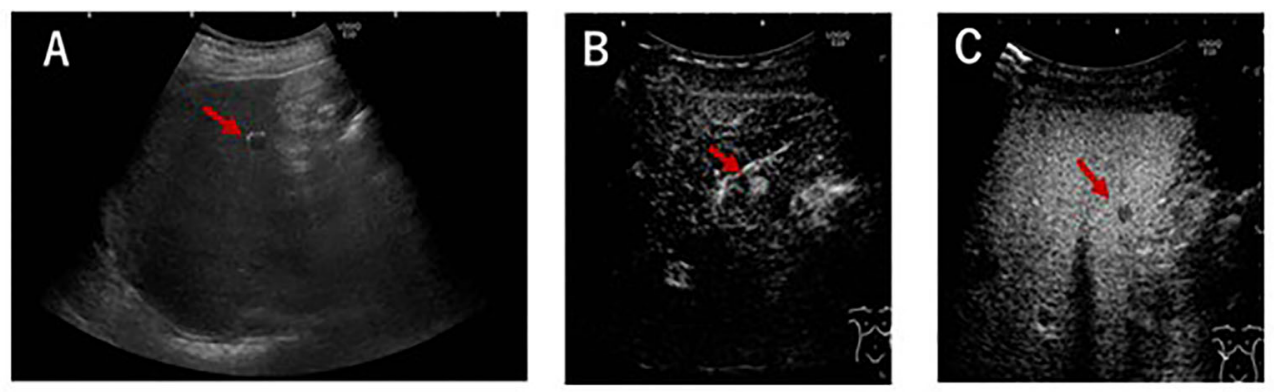

FIGURE 2 | US findings. (A) B-mode US, showing tiny hypoechoic nodules. (B, C) Sonazoid enhanced US during (B) early and (C) delayed vascular phases. A perfusion defect was also observed during the delayed phase.

pigmentation (Figure 3A). Pathological evaluation showed proliferation of abnormally pigmented tumor cells (Figure 3B), which were positive for Melan-A (Figure 3C), HMB45 and S100 (data not shown), confirming that these nodules were hepatic metastases of uveal melanoma. The highly proliferative nature and aggressive phenotype of these tumor cells were confirmed by high MIB-1 expression (Figure 3D). Immunohistochemical staining also showed infiltration of $\mathrm{CD} 8$, but not $\mathrm{CD} 4$, positive lymphocytes into metastatic nodule (Figures $3 \mathbf{E}-\mathbf{H}$ ). The number of CD8 positive lymphocytes was increased in the metastatic tumor as compared with non-tumor area and that of CD4 (Figure 3I).

Systemic chemotherapy with immune checkpoint inhibitors was proposed to the patient, but both he and his family refused aggressive treatment. The patient has been followed-up by CT and MRI at intervals of several months. He has experienced no
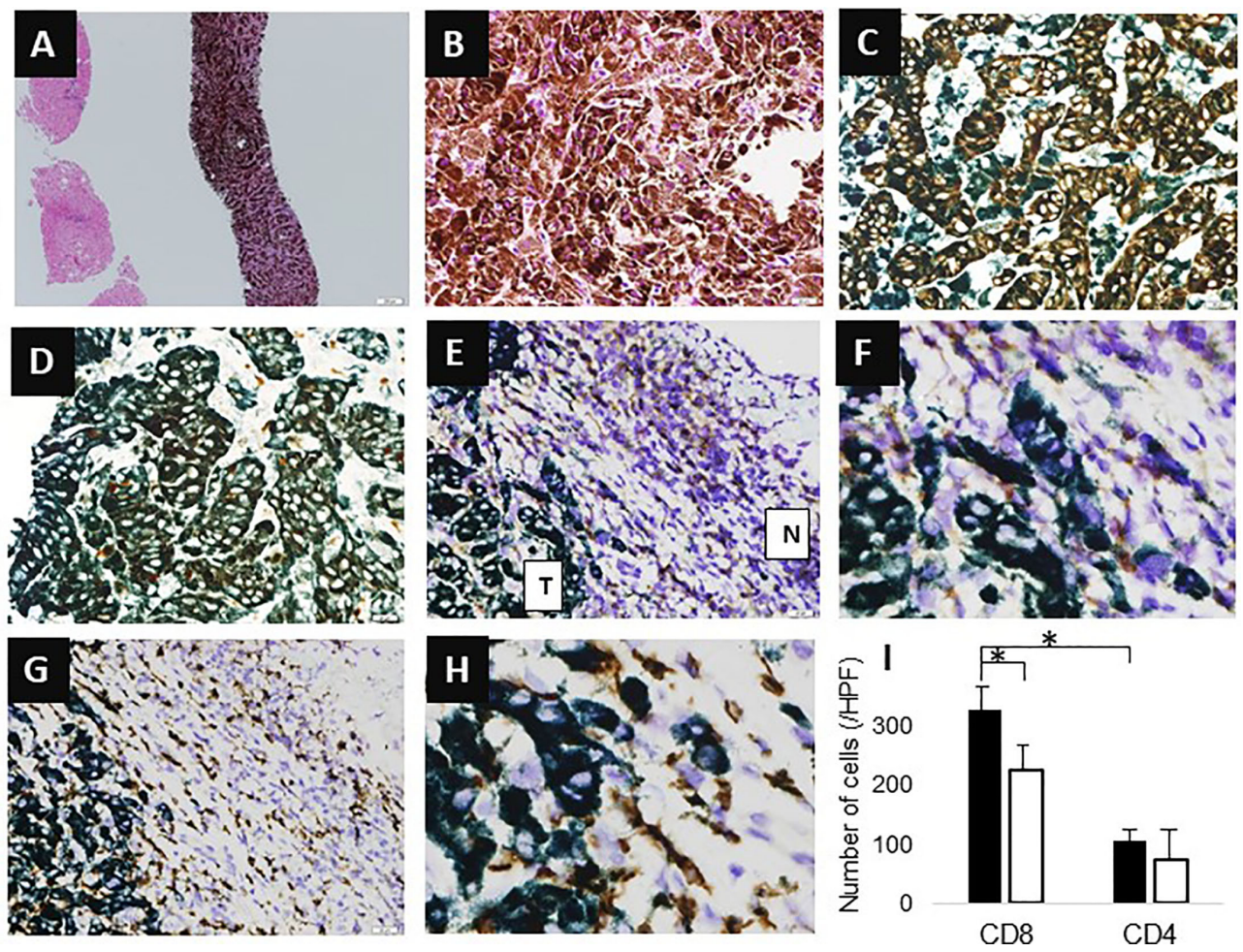

FIGURE 3 | Histological findings. (A, B) Hematoxylin-eosin (HE) staining (A, X4; B, X200). (C) Melan-A staining (X400). (D) MIB-1 staining (X400). (E, F) CD4 staining (E, X100; F, X400); T indicates tumor area, N indicates non-tumor area. (G, H) CD8 staining (G, X100; $\mathbf{H}, \mathbf{X} 400)$. (I) Number of infiltrating cells. Y-axis represents the number of infiltrating cells. The average number of cells in three high power fields is shown. Black bar means the number of cells in tumor area, whereas white bar means that of non-tumor area. Asterisk indicates statistical significance $(p<0.05)$. 
change in the size or radiological pattern of these nodules for 1 year after presentation (Figure 4). He remains asymptomatic and remains in good condition without any treatment.

\section{DISCUSSION}

The present study describes a patient diagnosed with confirmed liver metastases of uveal melanoma. Despite receiving no treatment, this patient has remained asymptomatic, with no change in the size of the metastatic nodules for at least 1 year after presentation. Although the prognosis of patients with metastases of uveal melanoma is usually limited, adequate host immune response by CD8 positive lymphocytes might suppress disease progression.

FDG-PET/CT was inadequate in the early diagnosis of liver metastases in the present patient. Liver metastases of uveal melanoma were reported to be less sensitive to detection by FDGPET/CT than liver metastases of cutaneous melanoma (13). Furthermore, FDG-PET/CT is relatively insensitive in the detection of small liver metastases (14), and the metastatic nodules in this patient were quite small, being only a few millimeters in diameter. Liver metastases of uveal melanoma have been reported to be hypodense on CT examination and to be enhanced with contrast, but these features are not specific to melanoma. Furthermore, small metastases are difficult to detect on CT examination (15).

Liver metastases often show high intensity on T1-weighted and diffusion-weighted MRI, various enhancement patterns on enhanced MRI and hypo-intensity on hepatobiliary phase (1619). High-intensity on T1-weighted images of metastatic melanoma is thought to reflect intratumoral hemorrhage or certain sized areas of melanin pigmentation (16). However, only melanin pigmentation could induce high T1 intensity in the small metastases observed in the present patient, weakening its ability to diagnose metastatic lesions in the hepatobiliary phase of EOB-MRI. Liver metastases are hypo- or iso-echoic on $B$ mode US, whereas contrast enhanced US shows strong enhancement in early arterial phase and is hypoechoic in delayed phase $(14,20)$. US and Sonazoid-enhanced US were effective for the detection and diagnosis of such small metastases in the present patient. Taken together, these findings indicate that MRI and contrast-enhanced US are useful for the detection and diagnosis of small liver metastases of malignant melanoma.

Effective systemic treatments for metastatic uveal melanoma have not been determined to date (20). Immunotherapy for uveal melanoma has been reported to be insufficient due to immune tolerance and low mutational burden $(21,22)$. The expression of programmed cell death ligand- 1 is known to be associated with the infiltration of lymphocytes to the tumor and patient outcome (23). Immunosuppressive regulatory $\mathrm{T}$ cells and myeloid-derived suppressor cells reduce cell-mediated immunity in patients with liver metastases of uveal melanoma (22). Moreover, liver metastases induce resistance to immunotherapy through the depletion of CD8 positive $\mathrm{T}$ lymphocytes via macrophages (24). CD8 positive T lymphocytes in patients with advanced metastatic uveal melanoma have been detected only in peritumoral lesions, not but within tumors (25), reducing the efficacy of systemic treatment with immune checkpoint inhibitors. The liver metastases in the present case remained stable for 1 year after presentation, or 2 years after first detection on follow-up CT. Pathologic examination showed the infiltration of CD8 positive lymphocytes into these metastatic lesions.

Correlations between tumor infiltration by CD8 positive $\mathrm{T}$ lymphocytes and patient prognosis have been reported in

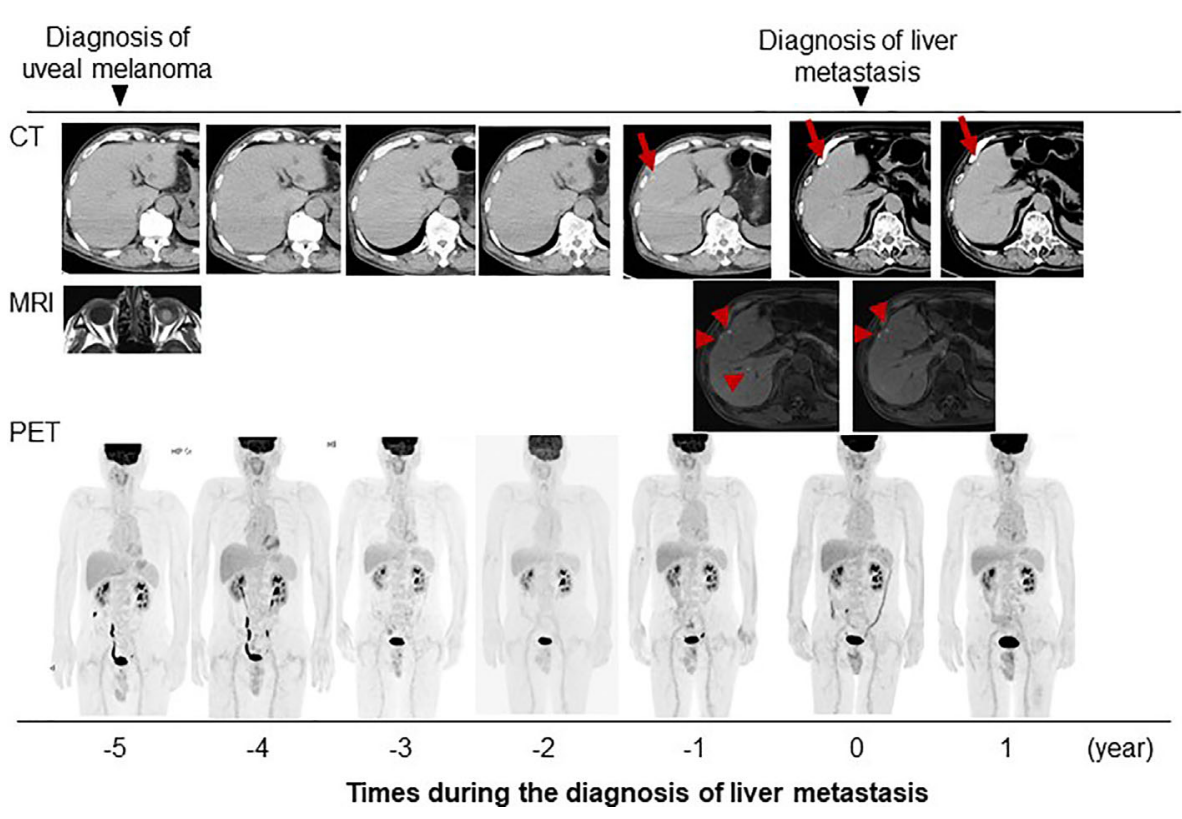

FIGURE 4 | Clinical course of present case with images. Red arrow and arrow head indicate the metastatic tumors detectable with imaging studies. 
patients with other types of malignant tumors (26, 27). For example, tumor infiltration by CD8 positive T lymphocytes was found to be significantly associated with disease-free survival and overall survival in breast cancer patients (26). CD8 positive $\mathrm{T}$ lymphocytes are thought to suppress micro-metastases in patients with colon cancer (27). Furthermore, intra-tumoral infiltration by CD8-positive, but not CD4-positive, lymphocyte has been associated with the prognosis and response to treatment in patients with malignant melanoma (28). The clinical course and pathology of the present patient suggest that the host immune response, including infiltration by CD8 lymphocytes, could suppress the progression of small-sized tumors. It is unclear when and why this immune suppression of metastatic tumors is disabled and these tumors progress. Immunotherapy may have shown a favorable anti-tumor effect in the present patient. Further careful observation of the present patient including molecular analysis of infiltrating CD8 lymphocytes, analysis of other immune cells such as NK cells or tumor microenvironment may provide clues to the optimal treatment of hepatic metastases of uveal melanoma.

In conclusion, this study described a patient with small hepatic metastases of uveal melanoma detected 5 years after primary tumor resection. MRI and contrast enhanced US could be useful in the early detection of hepatic metastases. This study also found that the hepatic metastases in the present patient were infiltrated by CD8 positive lymphocytes, and that, despite the absence of treatment, these metastases did not progress for 2 years. Although hepatic metastases of uveal melanoma have

\section{REFERENCES}

1. Kivela T. The Epidemiological Challenge of the Most Frequent Eye Cancer: Retinoblastoma, An Issue of Birth and Death. Br J Ophthalmol (2009) 93:1129-31. doi: 10.1136/bjo.2008.150292

2. Notting IC, Buijs JT, Que I, Mintardjo RE, van der Horst G, Karperien M, et al. Whole-Body Bioluminescent Imaging of Human Uveal Melanoma in a New Mouse Model of Local Tumor Growth and Metastasis. Invest Ophthalmol Vis Sci (2005) 46:1581-7. doi: 10.1167/iovs.04-0245

3. Kath R, Hayungs J, Bornfeld N, Sauerwein W, Hoffken K, Seeber S. Prognosis and Treatment of Disseminated Uveal Melanoma. Cancer (1993) 72:2219-23. doi: 10.1002/1097-0142(19931001)72:7<2219::AID-CNCR2820720725>3.0.CO;2-J

4. Rietschel P, Panageas KS, Hanlon C, Patel A, Abramson DH, Chapman PB. Variates of Survival in Metastatic Uveal Melanoma. J Clin Oncol (2005) 23:8076-80. doi: 10.1200/JCO.2005.02.6534

5. Collaborative Ocular Melanoma Study G. Assessment of Metastatic Disease Status At Death in 435 Patients With Large Choroidal Melanoma in the Collaborative Ocular Melanoma Study (Coms): COMS Report No. 15. Arch Ophthalmol (2001) 119:670-6. doi: 10.1001/archopht.119.5.670

6. Borthwick NJ, Thombs J, Polak M, Gabriel FG, Hungerford JL, Damato B, et al. The Biology of Micrometastases From Uveal Melanoma. J Clin Pathol (2011) 64:666-71. doi: 10.1136/jcp.2010.087999

7. Grossniklaus HE, Zhang Q, You S, McCarthy C, Heegaard S, Coupland SE. Metastatic Ocular Melanoma to the Liver Exhibits Infiltrative and Nodular Growth Patterns. Hum Pathol (2016) 57:165-75. doi: 10.1016/ j.humpath.2016.07.012

8. Javed A, Milhem M. Role of Natural Killer Cells in Uveal Melanoma. Cancers (Basel) (2020) 12:3694. doi: 10.3390/cancers12123694

9. Lorenzo D, Piulats JM, Ochoa M, Arias L, Gutierrez C, Catala J, et al. Clinical Predictors of Survival in Metastatic Uveal Melanoma. Jpn J Ophthalmol (2019) 63:197-209. doi: 10.1007/s10384-019-00656-9 shown insufficient response to immunotherapy, host immunity may be sufficient.

\section{DATA AVAILABILITY STATEMENT}

The original contributions presented in the study are included in the article/supplementary material. Further inquiries can be directed to the corresponding author.

\section{ETHICS STATEMENT}

Ethical review and approval was not required for the study on human participants in accordance with the local legislation and institutional requirements. The patients/participants provided their written informed consent to participate in this study.

\section{AUTHOR CONTRIBUTIONS}

KT designed the report. NT and KT wrote the manuscript. KT and KK collected the patient's clinical data. KT was responsible for the conception and revision of the manuscript. KT and KK carried out the clinical management of the patient. ST performed pathological evaluation. IY organized this work. All authors contributed to the article and approved the submitted version.

10. Diener-West M, Reynolds SM, Agugliaro DJ, Caldwell R, Cumming K, Earle JD, et al. Development of Metastatic Disease After Enrollment in the COMS Trials for Treatment of Choroidal Melanoma: Collaborative Ocular Melanoma Study Group Report No. 26. Arch Ophthalmol (2005) 123:163943. doi: 10.1001/archopht.123.12.1639

11. Aronow ME, Topham AK, Singh AD. Uveal Melanoma: 5-Year Update on Incidence, Treatment, and Survival (Seer 1973-2013). Ocul Oncol Pathol (2018) 4:145-51. doi: 10.1159/000480640

12. Durante MA, Rodriguez DA, Kurtenbach S, Kuznetsov JN, Sanchez MI, Decatur CL, et al. Single-Cell Analysis Reveals New Evolutionary Complexity in Uveal Melanoma. Nat Commun (2020) 11:496. doi: 10.1038/s41467-01914256-1

13. Strobel K, Bode B, Dummer R, Veit-Haibach P, Fischer DR, Imhof L, et al. Limited Value of 18F-FDG PET/CT and S-100B Tumour Marker in the Detection of Liver Metastases From Uveal Melanoma Compared to Liver Metastases From Cutaneous Melanoma. Eur J Nucl Med Mol Imaging (2009) 36:1774-82. doi: 10.1007/s00259-009-1175-0

14. Marshall E, Romaniuk C, Ghaneh P, Wong H, McKay M, Chopra M, et al. MRI in the Detection of Hepatic Metastases From High-Risk Uveal Melanoma: A Prospective Study in 188 Patients. Br J Ophthalmol (2013) 97:159-63. doi: 10.1136/bjophthalmol-2012-302323

15. Patel M, Winston CB, Marr BP, Carvajal RD, Schwartz GK, Wolchok J, et al. Characterization of Computed Tomography Scan Abnormalities in Patients With Biopsy-Proven Hepatic Metastases From Uveal Melanoma. Arch Ophthalmol (2011) 129:1576-82. doi: 10.1001/archophthalmol.2011.263

16. Balasubramanya R, Selvarajan SK, Cox M, Joshi G, Deshmukh S, Mitchell DG, et al. Imaging of Ocular Melanoma Metastasis. Br J Radiol (2016) 89:20160092. doi: 10.1259/bjr.20160092

17. Wagner M, Mariani P, Bidard FC, Rodrigues MJ, Farkhondeh F, Cassoux N, et al. Diffusion-Weighted MRI for Uveal Melanoma Liver Metastasis Detection. Eur Radiol (2015) 25:2263-73. doi: 10.1007/s00330-015-3662-y 
18. De Toni EN, Gallmeier E, Auernhammer CJ, Clevert DA. Contrast-Enhanced Ultrasound for Surveillance of Choroidal Carcinoma Patients: Features of Liver Metastasis Arising Several Years After Treatment of the Primary Tumor. Case Rep Oncol (2011) 4:336-42. doi: 10.1159/000329453

19. Choudhary MM, Gupta A, Bena J, Emch T, Singh AD. Hepatic Ultrasonography for Surveillance in Patients With Uveal Melanoma. JAMA Ophthalmol (2016) 134:174-80. doi: 10.1001/jamaophthalmol.2015.4810

20. Rantala ES, Hernberg M, Kivela TT. Overall Survival After Treatment for Metastatic Uveal Melanoma: A Systematic Review and Meta-Analysis. Melanoma Res (2019) 29:561-8. doi: 10.1097/CMR.0000000000000575

21. Johnson DB, Bao R, Ancell KK, Daniels AB, Wallace D, Sosman JA, et al. Response to Anti-PD-1 in Uveal Melanoma Without High-Volume Liver Metastasis. J Natl Compr Canc Netw (2019) 17:114-7. doi: 10.6004/ jnccn.2018.7070

22. Javed A, Arguello D, Johnston C, Gatalica Z, Terai M, Weight RM, et al. Pd-L1 Expression in Tumor Metastasis Is Different Between Uveal Melanoma and Cutaneous Melanoma. Immunotherapy (2017) 9:1323-30. doi: 10.2217/imt2017-0066

23. Zoroquiain P, Esposito E, Logan P, Aldrees S, Dias AB, Mansure JJ, et al. Programmed Cell Death Ligand-1 Expression in Tumor and Immune Cells is Associated With Better Patient Outcome and Decreased Tumor-Infiltrating Lymphocytes in Uveal Melanoma. Mod Pathol (2018) 31:1201-10. doi: 10.1038/s41379-018-0043-5

24. Yu J, Green MD, Li S, Sun Y, Journey SN, Choi JE, et al. Liver Metastasis Restrains Immunotherapy Efficacy Via Macrophage-Mediated T Cell Elimination. Nat Med (2021) 27:152-64. doi: 10.1038/s41591-020-1131-x
25. Krishna Y, McCarthy C, Kalirai H, Coupland SE. Inflammatory Cell Infiltrates in Advanced Metastatic Uveal Melanoma. Hum Pathol (2017) 66:159-66. doi: 10.1016/j.humpath.2017.06.005

26. Chen Z, Chen X, Zhou E, Chen G, Qian K, Wu X, et al. Intratumoral CD8(+) Cytotoxic Lymphocyte is a Favorable Prognostic Marker in Node-Negative Breast Cancer. PloS One (2014) 9:e95475. doi: 10.1371/journal.pone.0095475

27. Chiba T, Ohtani H, Mizoi T, Naito Y, Sato E, Nagura H, et al. Intraepithelial CD8+ T-Cell-Count Becomes a Prognostic Factor After a Longer Follow-Up Period in Human Colorectal Carcinoma: Possible Association With Suppression of Micrometastasis. Br J Cancer (2004) 91:1711-7. doi 10.1038/sj.bjc.6602201

28. Maibach F, Sadozai H, Seyed Jafari SM, Hunger RE, Schenk M. TumorInfiltrating Lymphocytes and Their Prognostic Value in Cutaneous Melanoma. Front Immunol (2020) 11:2105. doi: 10.3389/fimmu.2020.02105

Conflict of Interest: The authors declare that the research was conducted in the absence of any commercial or financial relationships that could be construed as a potential conflict of interest.

Copyright (C 2021 Takahashi, Tajiri, Kagoyana, Tanaka and Yasuda. This is an openaccess article distributed under the terms of the Creative Commons Attribution License (CC BY). The use, distribution or reproduction in other forums is permitted, provided the original author(s) and the copyright owner(s) are credited and that the original publication in this journal is cited, in accordance with accepted academic practice. No use, distribution or reproduction is permitted which does not comply with these terms. 